\title{
World Nutrition Rio 2012
}

We are one year away from the world congress World Nutrition Rio 2012: knowledge policy action (WNRio2012), which will take place in Rio de Janeiro, Brazil, from April 27 to April 30, 2012. The event was organized by a partnership between the World Public Health Nutrition Association (WPHNA) and the Brazilian Association of Graduate Studies on Public Health (ABRASCO - Associação Brasileira de Pós-Graduação em Saúde Coletiva) and sponsored by many institutions. The main purpose of this event is to help to consolidate bridges among knowledge, politics and actions in the field of Food and Nutrition in Public Health (ANSC - Alimentação e Nutrição em Saúde Coletiva). Therefore, the event hopes to offer a space to bring people together, exchange ideas, and to promote the convergence of researchers, public policy managers, local professionals and activists who work in this field throughout the world.

From the scientific praxis standpoint, the congress assumes the presupposition that nutrition should be capable of integrating four approaches: the biological, social (which includes the psychological and cultural aspects involved in someone's diet), environmental and economic. This is the only way of contributing to our passage from a period where human activity is largely based on exploitation, production and consumption to one that values preservation, conservation and sustainability.

In order to achieve the purpose of promoting bridges among knowledge, politics and actions, and based on the abovementioned presupposition, the organization of the event is being structured in a way that promotes an encompassing debate on the macro determinants of food and nutrition in the contemporary context, the responses of public agencies to the main issues of the nutritional agenda (as well as the current challenges for this response to be effective and transformational) and the experiences and possibilities of civil sector participation. The success of this program will require the active involvement of many entities: individuals and organizations promoting and working in the area of culture, economics, human rights, communication, consumption, environment, and in many relevant areas associated with the food system and ANSC agenda.

WNRio2012 will have some striking characteristics, such as: a program that encourages interaction among the invited participants and other speakers through debates and dialogues in small groups, workshops that culminate with concrete proposals for action; technological innovation, with interactive online transmission of the plenary debates; the involvement and participation of young researchers, managers and activists in all stages of the organization of the event; the recognition of different forms of expression; knowledge about ANSC, for example, by offering spaces for works of different modalities (abstracts of scientific studies, reports of experiences, videos, among others); allowing the event to take place in an academic environment, the Universidade do Rio de Janeiro (UERJ); promotion of activities that prepare for the event, such as the publication of documents that subsidize the debates that will occur during the congress; and the guaranteed source of funds that will come from enrollments and support by public institutions, thus rendering the event completely independent of sectors that have a conflict of interest with ANSC. 
When Brazil was presented as a candidate to host WNRio2012, ABRASCO by its Food and Nutrition in Public Health work group, all partners who supported it understood that this was a unique opportunity to give visibility to the Brazilian experience (academically and through public policies and civil society actions). This would also give Brazilians the opportunity to interact with many people and organizations from around the world that work in this field.

The possibility of holding a congress that is not just another event, but a precious moment of consolidation of ANSC in Brazil and elsewhere, is in our hands. In this sense, we invite the people and institutions who work in this area to contribute for the World Nutrition Rio 2012, its organization and its developments to be indeed a very innovative experience. The Journal of Nutrition with its intense contribution to quality scientific production and to the debate and reflection of relevant themes in food and nutrition expresses its commitment to WNRio 2012 by disclosing it through this editorial.

Inês Rugani Ribeiro de Castro Executive Secretary of the World Nutrition Rio 2012 